\title{
Conformal Geometry of Hypersurfaces in Lorentz Space Forms
}

\author{
Tongzhu $\mathrm{Li}^{1}$ and Changxiong $\mathrm{Nie}^{2}$ \\ ${ }^{1}$ Department of Mathematics, Beijing Institute of Technology, Beijing 100081, China \\ ${ }^{2}$ Faculty of Mathematics and Computer Sciences, Hubei University, Wuhan 430062, China
}

Correspondence should be addressed to Tongzhu Li; litz@bit.edu.cn

Received 22 June 2013; Accepted 8 August 2013

Academic Editor: Anna Fino

Copyright (C) 2013 T. Li and C. Nie. This is an open access article distributed under the Creative Commons Attribution License, which permits unrestricted use, distribution, and reproduction in any medium, provided the original work is properly cited.

Let $x: M^{n} \rightarrow M_{1}^{n+1}(c)$ be a space-like hypersurface without umbilical points in the Lorentz space form $M_{1}^{n+1}(c)$. We define the conformal metric and the conformal second fundamental form on the hypersurface, which determines the hypersurface up to conformal transformation of $M_{1}^{n+1}(c)$. We calculate the Euler-Lagrange equations of the volume functional of the hypersurface with respect to the conformal metric, whose critical point is called a Willmore hypersurface, and we give a conformal characteristic of the hypersurfaces with constant mean curvature and constant scalar curvature. Finally, we prove that if the hypersurface $x$ with constant mean curvature and constant scalar curvature is Willmore, then $x$ is a hypersurface in $H_{1}^{n+1}(-1)$.

\section{Introduction}

Let $x: M^{n} \rightarrow S^{n+p}$ be an immersed submanifold in sphere $S^{n+k}$. In [1], on the submanifold the Wang has constructed a complete invariant system of the Möbius transformation group of $S^{n+p}$. Especially for the hypersurface, the Möbius invariants, the Möbius metric, and the Möbius second fundamental form determine the hypersurface up to Möbius transformations provided the dimension of hypersurface $n \geq$ 3 (also see [2]). After that, the study of the Möbius geometry has been a topic of increasing interest (see [3-6]).

In this paper we study space-like hypersurfaces in the Lorentz space form $M_{1}^{n+1}(c)$ under the conformal transformation group. We follow Wang's idea and construct conformal invariants of space-like hypersurfaces which determine hypersurfaces up to a conformal transformation.

For the Lorentz space form, there exists a united conformal compactification $\mathbb{Q}_{1}^{n+1}$, which is the projectivized light cone in $\mathbb{R} P^{n+2}$ induced from $R_{2}^{n+3}$ (see $[7,8]$ ). Using conformal compactification $\mathbb{Q}_{1}^{n+1}$, we define the conformal metric $g$ and the conformal second fundamental form on a hypersurface in the Lorentz space form, which determines a hypersurface up to a conformal transformation. Clearly, the volume functional with respect to the conformal metric is a conformal invariant. We call a critical hypersurface of the volume functional Willmore hypersurface. There are many studies about the Willmore hypersurface in the Lorentz space form (see, $[7,9,10])$.

Our main goal is to calculate the Euler-Lagrange equation for the volume functional by conformal invariants and to find some special Willmore hypersurfaces. We find that maximal hypersurfaces in Lorentz space form are not Willmore in general if the dimension $n \geq 3$. We give a conformal characteristic of the hypersurfaces with constant mean curvature and constant scalar curvature. By the conformal characteristic, we prove that if the hypersurfaces are Willmore, then the hypersurfaces must be in $H_{1}^{n+1}(-1)$. Thus, isoparametric hypersurfaces in $S_{1}^{n+1}(1)$ and $R_{1}^{n+1}$ are not Willmore.

We organize the paper as follows. In Section 2, we define the conformal invariants and give conformal congruent theorem of hypersurfaces in the Lorentz space form. In Section 3, we calculate the Euler Lagrange equation for the volume functional. In Section 4, we give a conformal characteristic of space-like hypersurfaces with constant mean curvature and constant scalar curvature. In Section 5, we give some examples of the Willmore hypersurface and prove that some special hypersurfaces are not Willmore in general. 


\section{Conformal Invariants of Hypersurfaces in Lorentz Space}

In this section, we define some conformal invariants of hypersurface and give a congruent theorem of hypersurfaces under the conformal group of $M_{1}^{n+1}(c)$.

Let $R_{t}^{n+2}$ be the real vector space $R^{n+2}$ with the Lorenz inner product $\langle$,$\rangle given by$

$$
\langle X, Y\rangle:=-\sum_{i=1}^{t} x_{i} y_{i}+\sum_{j=t+1}^{n+2} x_{j} y_{j} .
$$

Let $M_{1}^{n+1}(c)$ be a Lorentz space form. When $c=0, M_{1}^{n+1}(c)=$ $R_{1}^{n+1}$; when $c=1, M_{1}^{n+1}(c)=S_{1}^{n+1}(1)$, and when $c=-1$, $M_{1}^{n+1}(c)=H_{1}^{n+1}(-1)$, where

$$
\begin{aligned}
S_{1}^{n+1}(1) & =\left\{x \in R_{1}^{n+2} \mid\langle x, x\rangle=1\right\}, \\
H_{1}^{n+1}(-1) & =\left\{x \in R_{2}^{n+2} \mid\langle x, x\rangle=-1\right\} .
\end{aligned}
$$

We denote by $C^{n+2}$ the cone in $\mathbb{R}_{2}^{n+3}$ and by $\mathbb{Q}_{1}^{n+1}$ the conformal compactification space in $\mathbb{R} P^{n+3}$ :

$$
\begin{gathered}
C^{n+2}:=\left\{X \in \mathbb{R}_{2}^{n+3} \mid\langle X, X\rangle=0, X \neq 0\right\}, \\
\mathbb{Q}_{1}^{n+1}:=\left\{[X] \in \mathbb{R} P^{n+2} \mid\langle X, X\rangle=0\right\} .
\end{gathered}
$$

Let $O(n+3,2)$ be the Lorentz group of $R_{2}^{n+3}$ keeping the Lorentz inner product $\langle$,$\rangle invariant. Then, O(n+3,2)$ is a transformation group on $\mathbb{Q}_{1}^{n+1}$ defined by

$$
T([X]):=[X T], \quad X \in C^{n+2}, T \in O(n+3,2) .
$$

Topologically, $\mathbb{Q}_{1}^{n+1}$ is identified with the compact space $S^{n} \times$ $S^{1} / S^{0}$, which is endowed by a standard Lorentz metric $h=$ $g_{S^{n}} \oplus\left(-g_{S^{1}}\right) \cdot \mathbb{Q}_{1}^{n+1}$ has conformal metric:

$$
[h]:=\left\{e^{\tau} h \mid \tau \in C^{\infty}\left(\mathbb{Q}_{1}^{n+1}\right)\right\},
$$

and $[O(n+3,2)]$ is the conformal transformation group of $\mathbb{Q}_{1}^{n+1}$.

We define the following mappings (without ambiguity all denote by $\sigma$ ):

$$
\begin{aligned}
\sigma: R_{1}^{n+1} & \longrightarrow \mathbb{Q}_{1}^{n+1}, \quad u \\
\sigma: S_{1}^{n+1}(1) & \longrightarrow \mathbb{Q}_{1}^{n+1}, \quad u \longmapsto[(1, u)], \\
\sigma: H_{1}^{n+1}(-1) & \longrightarrow \mathbb{Q}_{1}^{n+1}, \quad u \longmapsto[(u, 1)] .
\end{aligned}
$$

Using $\sigma$, we can regard hypersurfaces in $M_{1}^{n+1}(c)$ as submanifolds in $\mathbb{Q}_{1}^{n+1}$. A classical theorem states the following.

Theorem 1. Two hypersurfaces $x, \bar{x}: M^{n} \rightarrow M_{1}^{n+1}(c)$ are conformal equivalent if and only if there exists $T \in O(n+3,2)$ such that $\sigma \circ x=T(\sigma \circ \bar{x}): M^{n} \rightarrow \mathbb{Q}_{1}^{n+1}$.
Let $x: M^{n} \rightarrow M_{1}^{n+1}(c)$ be a space-like hypersurface without umbilical points; then, $(\sigma \circ x) *\left(T M^{n}\right)$ is a positive definite subbundle of $T \mathbb{Q}_{1}^{n+1}$. For any local lift $Z$ of the standard projection $\pi: C^{n+2} \rightarrow \mathbb{Q}_{1}^{n+1}$, we get a local lift $y:=Z \circ \sigma \circ x: U \rightarrow C^{n+1}$ of $\sigma \circ x: M \rightarrow \mathbb{Q}_{1}^{n+1}$, which is defined in an open subset $U$ of $M^{n}$. Thus, $\langle d y, d y\rangle=$ $\lambda^{2} d x \cdot d x$ is a local metric, which is conformal to the induced metric $d x \cdot d x$. We denote by $\Delta$ and $\kappa$ the Laplacian operator and the normalized scalar curvature with respect to the local positive definite metric $\langle d y, d y\rangle$, respectively. Then, we have the following.

Theorem 2. Let $x: M^{n} \rightarrow M_{1}^{n+1}(c)$ be a space-like hypersurface; then the 2-form $g:=-\left(\langle\Delta y, \Delta y\rangle-n^{2} \kappa\right)\langle d y, d y\rangle$ is a globally defined conformal invariant. Moreover, $g$ is positive definite at any nonumbilical point of $M^{n}$.

Proof. First, we prove that $g$ is well defined. Suppose that $y$ : $U \rightarrow C^{n+1}, \tilde{y}: \widetilde{U} \rightarrow C^{n+2}$ are different lifts defined in open subsets $U$ and $\widetilde{U}$ of $M$. For the local positive definite metrics $\langle,\rangle_{y}=\langle d y, d y\rangle$, we denote by $\Delta$ the Laplace operator, by $\nabla f$ the gradient of a function $f$ and by $\kappa$ the normalized scalar curvatures with respect to $\langle,\rangle_{y}$, respectively. Analogously for $\langle d \widetilde{y}, d \widetilde{y}\rangle$, we denote by $\widetilde{\Delta}$ the Laplace operator and by $\widetilde{\kappa}$ the normalized scalar curvatures. On $U \cap \widetilde{U}$, we have $\tilde{y}=e^{\tau} y$, where $\tau \in C^{\infty}(U \cap \widetilde{U})$. Therefore, $\langle d \tilde{y}, d \tilde{y}\rangle=e^{2 \tau}\langle d y, d y\rangle$. By some computations, we have

$$
\begin{gathered}
e^{2 \tau} \tilde{\Delta} f=\Delta f+(n-2)\langle\nabla \tau, \nabla f\rangle_{y}, \\
e^{2 \tau} \widetilde{\kappa}=\kappa-\frac{2}{m} \Delta \tau-\frac{n-2}{n}\langle\nabla \tau, \nabla \tau\rangle_{y} .
\end{gathered}
$$

Using these formula, it follows that

$$
\left(\langle\Delta y, \Delta y\rangle-n^{2} \kappa\right)\langle d y, d y\rangle=\left(\langle\widetilde{\Delta} \tilde{y}, \tilde{\Delta} \tilde{y}\rangle-n^{2} \tilde{\kappa}\right)\langle d \tilde{y}, d \tilde{y}\rangle .
$$

Next, we prove that $g$ is invariant under conformal transformations of $M_{1}^{n+1}(c)$. Let $\phi$ be a conformal transformation of $M_{1}^{n+1}(c)$, and we denote $\bar{x}=\phi(x)$; then, there is a $T \in O(n+$ $3,2)$ acting on $\mathbb{Q}_{1}^{n+1}$, and $y: U \rightarrow C^{n+2}$ is a lift of $\sigma \circ x: M \rightarrow$ $\mathbb{Q}_{1}^{n+1}$ defined in open subsets $U$; then, the submanifold $\bar{x}=$ $\phi(x)$ must have a local lift like $\bar{y}=e^{\tau} y T$. Since $T$ preserves the Lorentz inner product and the dilatation of the local lift $y$ will not impact the term $\left(\langle\Delta y, \Delta y\rangle-m^{2} \kappa\right)\langle d y, d y\rangle$, therefore the 2-form $g$ is invariant under conformal transformations. then

Now, let $M_{1}^{n+1}(c)=S_{1}^{n+1}(1)$ and take local lift $y=(1, x)$;

$$
-\left(\langle\Delta y, \Delta y\rangle-n^{2} \kappa\right)=\frac{n}{n-1}\left(|I I|^{2}-n H^{2}\right),
$$

where $I I$ and $H$ are the second fundamental form and the mean curvature of $x$, respectively. Thus, $g$ is positive definite at any nonumbilical point of $M^{n}$; analogously for hypersurfaces in $R_{1}^{n+1}$ and $H_{1}^{n+1}$. Thus; we complete the proof of Theorem 2. 
Now, we assume that space-like hypersurface $x: M^{n} \rightarrow$ $M_{1}^{n+1}(c)$ is umbilical-free; thus, the 2 -form $g$ is a positive definite. We call $g$ the conformal metric of hypersurface $x$. There exists a unique lift:

$$
Y: M \longrightarrow C^{n+2}
$$

such that $g=\langle d Y, d Y\rangle$. We call $Y$ the conformal position vector of $x$. Theorem 2 implies the following.

Theorem 3. Two space-like hypersurfaces $x, \bar{x}: M^{n} \rightarrow$ $M_{1}^{n+1}(c)$ are conformal equivalent if and only if there exists $T \in O(n+3,2)$ such that $\bar{Y}=Y T$, where $Y, \tilde{Y}$ are the conformal position vectors of $x, \bar{x}$, respectively.

Let $\left\{E_{1}, \ldots, E_{n}\right\}$ be a local orthonormal basis of $M^{n}$ with respect to $g$ with dual basis $\left\{\omega_{1}, \ldots, \omega_{n}\right\}$. Denote $Y_{i}=E_{i}(Y)$. We define

$$
N:=-\frac{1}{n} \Delta Y-\frac{1}{2 n^{2}}\langle\Delta Y, \Delta Y\rangle Y
$$

where $\Delta$ is the Laplace operator of $g$; then, we have

$$
\begin{gathered}
\langle N, Y\rangle=1, \quad\langle N, N\rangle=0, \quad\left\langle N, Y_{k}\right\rangle=0, \\
\left\langle Y_{i}, Y_{j}\right\rangle=\delta_{i j}, \quad 1 \leq k \leq n .
\end{gathered}
$$

We may decompose $R_{2}^{n+3}$ such that

$$
R_{2}^{n+3}=\operatorname{span}\{Y, N\} \oplus \operatorname{span}\left\{Y_{1}, \ldots, Y_{n}\right\} \oplus \mathbb{V}
$$

where $\mathbb{V} \perp \operatorname{span}\left\{Y, N, Y_{1}, \ldots, Y_{n}\right\}$. We call $\mathbb{V}$ the conformal normal bundle of $x$, which is linear bundle. Let $\xi$ be a local section of $\mathbb{V}$ and $\langle\xi, \xi\rangle=-1$; then, $\left\{Y, N, Y_{1}, \ldots, Y_{n}, \xi\right\}$ forms a moving frame in $R_{2}^{n+3}$ along $M^{n}$. We may write the structure equations as follows:

$$
\begin{gathered}
d Y=\sum_{i} \omega_{i} Y_{i}, \\
d N=\sum_{i} A_{i} Y_{i}+C \xi \\
d Y_{i}=-A_{i} Y-\omega_{i} N+\sum_{j} \omega_{i j} Y_{j}+B_{i} \xi, \\
d \xi=C Y+\sum_{i} B_{i} Y_{i},
\end{gathered}
$$

where $\left\{A_{i}, C, B_{i}, \omega_{i j}\right\}$ are 1 -forms on $M^{n}$ with $\omega_{i j}=-\omega_{i j}$. It is clear that $A:=\sum_{i} A_{i} \otimes \omega_{i}, B:=\sum_{i} B_{i} \otimes \omega_{i}, C$ are globally defined conformal invariants. We call $B$ the conformal second fundamental form, $A$ the conformal 2tensor, and $C$ conformal 1 -form, respectively. If we write

$$
A_{i}=\sum_{j} A_{i j} \omega_{j}, \quad B_{i}=\sum_{j} B_{i j} \omega_{j}, \quad C=\sum_{i} C_{i} \omega_{i},
$$

then we can define the covariant derivatives of these tensors and curvature tensor with respect to conformal metric $g$ :

$$
\begin{gathered}
\sum_{j} C_{i, j} \omega_{j}=d C_{i}+\sum_{k} C_{k} \omega_{k j}, \\
\sum_{k} A_{i j, k} \omega_{k}=d A_{i j}+\sum_{k} A_{i k} \omega_{k j}+\sum_{k} A_{k j} \omega_{k i}, \\
\sum_{k} B_{i j, k} \omega_{k}=d B_{i j}+\sum_{k} B_{i k} \omega_{k j}+\sum_{k} B_{k j} \omega_{k i} .
\end{gathered}
$$

By exterior differentiation of structure equations (14) and the definition of the covariant derivative of conformal invariants, we can get the integrable conditions of the structure equations:

$$
\begin{gathered}
A_{i j}=A_{j i}, \quad B_{i j}=B_{j i} \\
A_{i j, k}-A_{i k, j}=B_{i j} C_{k}-B_{i k} C_{j} \\
B_{i j, k}-B_{i k, j}=\delta_{i j} C_{k}-\delta_{i k} C_{j} \\
C_{i, j}-C_{j, i}=\sum_{k}\left(B_{i k} A_{k j}-B_{j k} A_{k i}\right) \\
R_{i j k l}=B_{i l} B_{j k}-B_{i k} B_{j l}+A_{i k} \delta_{j l}+A_{j l} \delta_{i k}-A_{i l} \delta_{j k}-A_{j k} \delta_{i l} .
\end{gathered}
$$

Since $g=\langle d Y, d Y\rangle$, we get

$$
\langle\Delta Y, \Delta Y\rangle=n^{2} \kappa-1
$$

From structure equation, we have

$$
\Delta Y=-\operatorname{tr}(A)-n N+\operatorname{tr}(B) \xi .
$$

Furthermore, we have

$$
\begin{gathered}
\operatorname{tr}(A)=\frac{1}{2 n}\left(n^{2} \kappa-1\right), \\
R_{i j}=\operatorname{tr}(A) \delta_{i j}+(n-2) A_{i j}+\sum_{k} B_{i k} B_{k j}, \\
(1-n) C_{i}=\sum_{j} B_{i j, j}, \quad \sum_{i j} B_{i j}^{2}=\frac{n-1}{n}, \quad \sum_{i} B_{i i}=0,
\end{gathered}
$$

where $\kappa$ is the normalized scalar curvature of $g$. From (24), we see that when $n \geq 3$, all coefficients in the structure equations are determined by the conformal metric $g$ and the conformal second fundamental form $B$; thus, we get the following conformal congruent theorem.

Theorem 4. Two space-like hypersurfaces $x: M^{n} \rightarrow$ $M_{1}^{n+1}(c)$ and $\bar{x}: M^{n} \rightarrow M_{1}^{n+1}(c)(n \geq 3)$ are conformal equivalent if and only if there exists a diffeomorphism $\varphi$ : $M^{n} \rightarrow M^{n}$ which preserves the conformal metric and the conformal second fundamental form.

Remark 5. By using the same method as in [1], we can define conformal invariants of space-like submanifold 
$x: M^{m} \rightarrow M_{1}^{n+1}(c)$. But for time-like submanifolds, the situation is very different. In fact, for space-like submanifolds, the globally defined conformal invariant $g:=-(\langle\Delta y, \Delta y\rangle-$ $\left.m^{2} \kappa\right)\langle d y, d y\rangle$ is positive definite or semipositive definite, and for time-like submanifolds, the globally defined conformal invariant $g:=-\left(\langle\Delta y, \Delta y\rangle-m^{2} \kappa\right)\langle d y, d y\rangle$ is non-definite.

Next, we give the relations between the conformal invariants and isometric invariants of $x: M^{n} \rightarrow M_{1}^{n+1}(c)$.

First, we consider space-like hypersurface in $R_{1}^{n+1}$. Let $x$ : $M^{n} \rightarrow R_{1}^{n+1}$ be a space-like hypersurface without umbilical points. Let $\left\{e_{1}, \ldots, e_{n}\right\}$ be an orthonormal local basis for the induced metric $I=\langle d x, d x\rangle$ with dual basis $\left\{\theta_{1}, \ldots, \theta_{n}\right\}$. Let $e_{n+1}$ be a normal vector field of $x$, and $\left\langle e_{n+1}, e_{n+1}\right\rangle=-1$. Then, we have the first and second fundamental forms $I, I I$ and the mean curvature $H$. We may write $I=\sum_{i} \theta_{i} \otimes \theta_{i}$; $I I=\sum_{i j} h_{i j} \theta_{i} \otimes \theta_{j} ; H=(1 / n) \sum_{i} h_{i i}$. Denote by $\Delta_{M}$ the Laplacian and $\kappa_{M}$ the normalized scalar curvature for $I$. By structure equation and Gauss equation of $x$ we get that

$$
\Delta_{M} x=n H e_{n+1}, \quad \kappa_{M}=\frac{-1}{n(n-1)}\left(n^{2}|H|^{2}-|I I|^{2}\right) .
$$

For $x: M^{n} \rightarrow R_{1}^{n+1}$, there is a lift:

$$
y: M^{n} \longrightarrow C^{n+2}, \quad y=\left(\frac{\langle x, x\rangle+1}{2}, x, \frac{\langle x, x\rangle-1}{2}\right) .
$$

So, we get

$$
\langle d y, d y\rangle=\langle d x, d x\rangle=I, \quad \Delta=\Delta_{M}, \quad \kappa=\kappa_{M} .
$$

It follows from (25) that

$$
\langle\Delta Y, \Delta Y\rangle-n^{2} \kappa=\frac{n}{n-1}\left(-|I I|^{2}+n|H|^{2}\right) .
$$

Therefore, the conformal metric and conformal position vector of $x$ are as follows:

$$
\begin{gathered}
g=\frac{n}{n-1}\left(|I I|^{2}-n|H|^{2}\right)\langle d x, d x\rangle:=e^{2 \tau} I \\
Y=\sqrt{\frac{n}{n-1}\left(|I I|^{2}-n|H|^{2}\right)}\left(\frac{\langle x, x\rangle+1}{2}, x, \frac{\langle x, x\rangle-1}{2}\right) .
\end{gathered}
$$

Let $E_{i}=e^{-\tau} e_{i}$; then $\left\{E_{i} \mid 1 \leq i \leq n\right\}$ are the local orthonormal basis for $g$, and with the dual basis $\omega_{i}=e^{\tau} \theta_{i}$. Let

$$
\begin{gathered}
y_{i}=\left(\left\langle x, e_{i}\right\rangle, e_{i},\left\langle x, e_{i}\right\rangle\right), \\
y_{n+1}=\left(\left\langle x, e_{n+1}\right\rangle, e_{n+1},\left\langle x, e_{n+1}\right\rangle\right) .
\end{gathered}
$$

By some calculations, we can obtain that

$$
\begin{gathered}
Y=e^{\tau} y, \quad Y_{i}=e^{\tau}\left(\tau_{i} y+y_{i}\right), \quad \xi=-H y+y_{n+1}, \\
-e^{\tau} N=\frac{1}{2}\left(|\nabla \tau|^{2}-|H|^{2}\right) y+\sum_{i} \tau_{i} y_{i}+H y_{n+1}+(1, \overrightarrow{0}, 1),
\end{gathered}
$$

where $\tau_{i}=e_{i}(\tau)$ and $|\nabla \tau|^{2}=\sum_{i} \tau_{i}^{2}$.
By a direct calculation, we get the following expression of the conformal invariants $A, B$, and $C$ :

$$
\begin{gathered}
A_{i j}=e^{-2 \tau}\left[\tau_{i} \tau_{j}-h_{i j} H-\tau_{i, j}+\frac{1}{2}\left(-|\nabla \tau|^{2}+|H|^{2}\right) \delta_{i j}\right], \\
B_{i j}=e^{-\tau}\left(h_{i j}-H \delta_{i j}\right), \\
C_{i}=e^{-2 \tau}\left(H \tau_{i}-H_{i}-\sum_{j} h_{i j} \tau_{j}\right)
\end{gathered}
$$

where $\tau_{i, j}$ is the Hessian of $\tau$ for $I$ and $H_{i}=e_{i}(H)$.

Using the same methods, we can obtain relations between the conformal invariants and isometric invariants of $x$ : $M^{n} \rightarrow S_{1}^{n+1}(1)$ and $x: M^{n} \rightarrow H_{1}^{n+1}(-1)$. We have the following unitied expression of the conformal invariants $A$, $B$, and $C$ :

$$
\begin{gathered}
A_{i j}=e^{-2 \tau}\left[\tau_{i} \tau_{j}-\tau_{i, j}-h_{i j} H+\frac{1}{2}\left(-|\nabla \tau|^{2}+|H|^{2}+\epsilon\right) \delta_{i j}\right], \\
B_{i j}=e^{-\tau}\left(h_{i j}-H \delta_{i j}\right), \\
C_{i}=e^{-2 \tau}\left(H \tau_{i}-H_{i}-\sum_{j} h_{i j} \tau_{j}\right),
\end{gathered}
$$

where $\epsilon=1$ for $x: M^{n} \rightarrow S_{1}^{n+1}(1)$ and $\epsilon=-1$ for $x: M^{n} \rightarrow$ $H_{1}^{n+1}(-1)$.

\section{The First Variation of the Conformal Volume Functional}

Let $x_{0}: M^{n} \rightarrow M_{1}^{n+1}(c)$ be a compact space-like hypersurface with boundary $\partial M^{n}$. We define the generalized Willmore functional $W\left(M^{n}\right)$ (as the volume functional of the conformal metric $g$ ):

$$
\begin{aligned}
& \operatorname{Vol}_{g}\left(M^{n}\right) \\
& \quad=\int_{M} d M_{g}=\left(\frac{n}{n-1}\right)^{n / 2} \int_{M^{n}}\left(|I I|^{2}-n H^{2}\right)^{n / 2} d M_{I} .
\end{aligned}
$$

A critical hypersurface of the conformal volume functional is called a Willmore hypersurface.

Let $x: M^{n} \times R \rightarrow M_{1}^{n+1}(c)$ be an admissible variation of $x_{0}$ such that

$$
\begin{gathered}
\left.x(\cdot, t)\right|_{\partial M^{n}}=\left.x_{0}\right|_{\partial M^{n}}, \\
\left.d x_{t}\left(T_{p} M^{n}\right)\right|_{\partial M^{n}}=\left.d x_{0}\left(T_{p} M^{n}\right)\right|_{\partial M^{n}},
\end{gathered}
$$

for each small $t$. For each $t, x_{t}$ has the conformal metric $g_{t}$. As in Section 2, we have a moving frame $\left\{Y, N, Y_{i}, \xi\right\}$ in $R_{2}^{n+3}$ and the Willmore functional $W\left(x_{t}\right)$. Let $\xi$ be a local basis for 
the conformal normal bundle $\mathbb{V}_{t}$ of $x_{t}$. Denote by $d$ and $d_{M}$ the differential operators on $M^{n} \times R$ and $M^{n}$, respectively. Then, we have

$$
d=d_{M}+d t \wedge \frac{\partial}{\partial t}
$$

By (31), we can find functions $w, v_{i}, v: M^{n} \times R \rightarrow R$ such that

$$
\frac{\partial Y}{\partial t}=w Y+\sum_{i} v_{i} Y_{i}+v \xi .
$$

Since $\left\{Y, N, Y_{i}, \xi\right\}$ is a moving frame along $M^{n} \times R$, it follows from (37) and (38) that

$$
\begin{gathered}
d Y=w d t Y+\sum_{i} \Omega_{i} Y_{i}+V \xi, \\
d N=-w d t N+\sum_{i} \Psi_{i} Y_{i}+\Phi \xi, \\
d Y_{i}=-\Psi_{i} Y-\Omega_{i} N+\sum_{j} \Omega_{i j} Y_{j}+F_{i} \xi, \\
d \xi=-\Phi Y-V N-\sum_{i} F_{i} Y_{i},
\end{gathered}
$$

where $\Omega_{i j}=-\Omega_{j i}, \Omega_{i}=\omega_{i}+v_{i} d t, V=v d t$. By exterior differentiation of (39), we get

$$
\begin{gathered}
d \Omega_{i}=\sum_{j} \Omega_{i j} \wedge \Omega_{j}+w d t \wedge \Omega_{i}+v d t \wedge F_{i} \\
d v \wedge d t=\sum_{i} \Omega_{i} \wedge F_{i} \\
d F_{i}=\sum_{j} F_{j} \wedge \Omega_{j i}-\Omega_{i} \wedge \Phi-\Psi_{i} \wedge V \\
d \Omega_{i j}=\sum_{k} \Omega_{i k} \wedge \Omega_{k j}-\Omega_{i} \wedge \Psi_{j}-\Psi_{i} \wedge \Omega_{j}+F_{i} \wedge F_{\mathrm{j}} .
\end{gathered}
$$

Since $T^{*}\left(M^{n} \times R\right)=T^{*} M^{n} \oplus T^{*} R$, we have the following decomposition:

$$
\begin{gathered}
\Omega_{i j}=\omega_{i j}+L_{i j} d t, \quad \Psi_{i}=A_{i}+u_{i} d t, \\
\Phi=C+u d t, \quad F_{i}=B_{i}+b_{i} d t,
\end{gathered}
$$

where $\left\{u_{i}, u, L_{i j}, b_{i}\right\}$ are local functions on $M \times \mathbb{R}$. Using (40) and comparing the terms in $T^{*} M \wedge d t$, we get

$$
\frac{\partial \omega_{i}}{\partial t}=\sum_{j}\left(v_{i, j}+L_{i j}+B_{i j} v\right) \omega_{j}+w \omega_{i},
$$

where $\left\{v_{i, j}\right\}$ is the covariant derivative of $\sum v_{i} E_{i}$ with respect to $g_{t}$. Here, we have used the notations of conformal invariants $\left\{A_{i j}, B_{i j}, C_{i}\right\}$ for $x_{t}$. By the same way, we get from (40) that

$$
\begin{gathered}
b_{i}=e_{i}(v)+\sum_{j} B_{i j} v_{j} \\
\frac{\partial F_{i}}{\partial t}=\sum_{j}\left(b_{i, j}+\sum_{k} L_{i k} B_{k j}+A_{i j} v-v_{i} C_{j}\right) \omega_{j}+u \omega_{i},
\end{gathered}
$$

where $\left\{b_{i, j}\right\}$ are covariant derivatives of $\sum_{i} b_{i} \omega_{i}$. Using (42) and (43), we get

$$
\begin{gathered}
\frac{\partial B_{i j}}{\partial t}+w B_{i j}=b_{i, j}+\sum_{k}\left(L_{i k} B_{i k}-B_{i k} L_{k j}\right)-v_{i} C_{j} \\
+A_{i j} v+u \delta_{i j}-\sum_{k} B_{i k}\left(v_{k, j}+B_{k j} v\right), \\
b_{i, j}=v_{, i j}+\sum_{k}\left(B_{i k, j} v_{k}+B_{i k} v_{k, j}\right), \\
v_{, i j}=d_{M} e_{i}(v)+\sum_{j} e_{j}(v) \omega_{j i} .
\end{gathered}
$$

Therefore, we have

$$
\begin{aligned}
& \frac{\partial B_{i j}}{\partial t}+w B_{i j}= v_{, i j}+\sum_{k}\left(L_{i k} B_{i k}-B_{i k} L_{k j}+B_{i j, k} v_{k}\right) \\
&+\left(A_{i j}-\sum_{k} B_{i k} B_{k j}\right)+\left(u-\sum_{k} v_{k} C_{k}\right) \delta_{i j} \\
& \frac{n-1}{n} w=\sum_{i j} B_{i j}\left[v_{i j}+\left(A_{i j}-\sum_{k} B_{i k} B_{k j}\right) v\right]
\end{aligned}
$$

Now, we calculate the first variation of the following conformal volume functional:

$$
W(t)=\operatorname{vol}\left(g_{t}\right)=\int_{M^{n}} \omega_{1} \wedge \cdots \wedge \omega_{n}=\int_{M^{n}} d M_{t},
$$

where $d M$ is the volume for $g_{t}$. From (42) and (46) we get $W^{\prime}(t)$

$$
\begin{aligned}
& =\sum_{i} \int_{M^{n}} \omega_{1} \wedge \cdots \wedge \frac{\partial \omega_{i}}{\partial t} \wedge \cdots \wedge \omega_{n}=n \int_{M^{n}} w d M_{t} \\
& =\frac{n^{2}}{n-1} \int_{M^{n}}\left\{\sum_{i j} B_{i j}\left[v_{, i j}+\left(A_{i j}-\sum_{k} B_{i k} B_{k j}\right) v\right]\right\} d M_{t} .
\end{aligned}
$$

From the fact that the variation is admissible, we know that $v_{i}=0, v=0$ and $e_{i}(v)=0$ on $\partial M^{n}$. It follows from (48) and Green's formula that

$$
\begin{aligned}
W^{\prime}(t)= & \frac{n^{2}}{n-1} \\
& \times \int_{M^{n}}\left\{\sum_{i j} B_{i j, i j}+\sum_{i j} A_{i j} B_{i j}-\sum_{i j k} B_{i k} B_{k j} B_{i j}\right\} \\
& \times v d M_{t} .
\end{aligned}
$$

Thus, we have the following theorem. 
Theorem 6. A space-like hypersurface $x: M^{n} \rightarrow M_{1}^{n+1}(c)$ is a Willmore hypersurface (i.e., a critical hypersurface to the conformal volume functional) if and only if

$$
\sum_{i j} B_{i j, i j}+\sum_{i j} A_{i j} B_{i j}-\sum_{i j k} B_{i k} B_{k j} B_{i j}=0 .
$$

Using (24), we can write the Euler-Lagrange equations as

$$
\sum_{i} C_{i, i}+\sum_{i j}\left(\frac{1}{n-1} R_{i j}-A_{i j}\right) B_{i j}=0 .
$$

Theorem 7. Any maximal (or zero mean curvature) space-like surface in Lorentz space forms is a Willmore surface.

Proof. Let $x: M^{2} \rightarrow R_{1}^{3}$ be a space-like surface. Let $\left\{e_{1}, e_{2}\right\}$ be a local orthonormal basis of $\langle d x, d x\rangle$ and $e_{3}$ a local normal vector field. From (32), we get that

$$
\sum_{i j} A_{i j} B_{i j}=\sum_{i j} e^{-2 \tau} B_{i j}\left(\tau_{i} \tau_{j}-\tau_{i, j}\right)+\sum_{i j} e^{-\tau} B_{i j} B_{i j} H .
$$

Since we have the following relations of connections:

$$
\omega_{i j}=\theta_{i j}+\tau_{i} \omega_{j}-\tau_{j} \omega_{i},
$$

a direct calculation implies that

$$
\sum_{i} C_{i, i}=\sum_{i j} e^{-2 \tau} B_{i j}\left(\tau_{i} \tau_{j}-\tau_{i, j}\right)-e^{-3 \tau} \Delta_{M} H .
$$

From (51), we have

$$
\sum_{i} C_{i, i}-\sum_{i j} A_{i j} B_{i j}=-e^{-3 \tau}\left(\Delta_{M} H+e^{2 \tau} \sum_{i j} B_{i j} B_{i j} H\right) .
$$

If $x$ is a maximal space-like surface, that is, $H=0$ and $R_{i j}=$ $\kappa \delta_{i j}$, thus $x$ is Willmore.

Similarly, we can verify that maximal space-like surfaces in $S_{1}^{3}(1)$ and $H_{1}^{3}(-1)$ are also Willmore. Thus, we complete the proof of Theorem 7 .

\section{A Characteristic of CMC Hypersurfaces and}

$$
\kappa_{M}=\text { Constant }
$$

In this section, we consider space-like hypersurfaces with constant mean curvature and constant scalar curvature.

Proposition 8. Let $x: M^{n} \rightarrow M_{1}^{n+1}(c)$ be a space-like hypersurface without umbilical points. If the mean curvature and scalar curvature of $x$ are constant, then conformal invariants of $x$ satisfy

$$
\text { (1), } C=0, \quad(2), A=\mu B+\lambda g,
$$

where $\mu, \lambda$ are constant.

Proof. First, we consider the space-like hypersurface $x$ : $M^{n} \rightarrow R_{1}^{n+1}$. Since $H$ and $\kappa$ are constant, then by the Gauss equation we have that

$$
e^{2 \tau}=\frac{n}{n-1}\left(|I I|^{2}-n H^{2}\right)=\text { constant. }
$$

From (32), we get that

$$
\begin{gathered}
C_{i}=0, \quad B_{i j}=e^{-\tau}\left(h_{i j}-H \delta_{i j}\right), \\
A_{i j}=e^{-2 \tau}\left[\frac{1}{2} H^{2}-H h_{i j}\right] .
\end{gathered}
$$

From (58), Let $\mu=-e^{-\tau} H$ and $\lambda=-(1 / 2) e^{-2 \tau} H^{2}$; then, we prove the formula (56).

Similarly, we can prove the formula (56) for $M_{1}^{n+1}(c)=$ $S_{1}^{n+1}(1)$ and $M_{1}^{n+1}(c)=H_{1}^{n+1}(-1)$. Thus, we complete the proof of Proposition 8.

Theorem 9. Let $x: M^{n} \rightarrow M_{1}^{n+1}(c)$ be a space-like hypersurface without umbilical points. If conformal invariants of $x$ satisfy

$$
\text { (1), } C=0, \quad(2), A=\mu B+\lambda g .
$$

Then $x$ is conformal equivalent to a space-like hypersurface with constant mean curvature and constant scalar curvature.

Proof. Since $C=0$, from (20), we can take the local orthonormal basis $\left\{E_{1}, \ldots, E_{n}\right\}$ such that

$$
\left(B_{i j}\right)=\operatorname{diag}\left(b_{1}, \ldots, b_{n}\right), \quad\left(A_{i j}\right)=\operatorname{diag}\left(a_{1}, \ldots, a_{n}\right) .
$$

Since $A=\mu B+\lambda g$, from structure equations (14) we get that

$$
d N-\lambda d Y-\mu d \xi=0 .
$$

Taking exterior differentiation of (61), we get that

$$
d \lambda \wedge d Y+d \mu \wedge d \xi=0 .
$$

Writing $d \lambda=\sum_{i} \lambda_{i} \omega_{i}, d \mu=\sum_{i} \mu_{i} \omega_{i}$, from (62) we get that

$$
\sum_{i j k}\left(\lambda_{k} \delta_{i j}+\mu_{k} B_{i j}\right) \omega_{k} \wedge \omega_{j} Y_{i}=0,
$$

which implies that

$$
\lambda_{k} \delta_{i j}+\mu_{k} B_{i j}=\lambda_{j} \delta_{i k}+\mu_{j} B_{i k} .
$$

Set $i=j$ in (64) and taking summation over $i$, we get that

$$
\lambda_{k}-\frac{1}{n-1} \mu_{k} b_{k}=0 .
$$

Set $i=j \neq k$ in (65), from (60), we get that

$$
\lambda_{k}+\mu_{k} b_{i}=0, \quad i \neq k .
$$

Combining (65) and (66), we get that

$$
\mu_{k}\left(b_{i}+\frac{1}{n-1} b_{k}\right)=0, \quad i \neq k .
$$

If $\mu_{i}=0,1 \leq i \leq n$, then $\lambda_{i}=0,1 \leq i \leq n$.

If $\mu_{i} \neq 0$, for some $i$; we can assume that $\mu_{1} \neq 0$. Then, from (67), we have

$$
b_{i}=-\frac{1}{n-1} b_{1}, \quad i \neq 1
$$


Since $\operatorname{tr}(B)=0$ and $|B|^{2}=(n-1) / n$, thus we have

$$
b_{1}=\frac{n-1}{n}, \quad b_{2}=\cdots=b_{n}=\frac{-1}{n} .
$$

Using $d B_{i j}+\sum_{k} B_{k j} \omega_{k i}+\sum_{k} B_{i k} \omega_{k j}=\sum_{k} B_{i j, k} \omega_{k}$ and (69), we get that

$$
\omega_{1 i}=0, \quad 1<i \leq n .
$$

Thus, we have

$$
R_{1 i 1 i}=0, \quad 1<i \leq n, \quad a_{2}=\cdots=a_{n} .
$$

Since $a_{i}=\mu b_{i}+\lambda$, then from (71), we have

$$
\begin{aligned}
& 2 \lambda+\frac{n-2}{n} \mu=\frac{n-1}{n^{2}}, \\
& 2 \lambda_{1}+\frac{n-2}{n} \mu_{1}=0 .
\end{aligned}
$$

From (66), we have

$$
\lambda_{1}+\frac{1}{n} \mu_{1}=0
$$

From (73) and (74), we get that

$$
\mu_{1}=0
$$

This is a contradiction; so $\mu_{i}=0,1 \leq i \leq n$. Since $\mu_{i}=0$, $\lambda_{i}=0,1 \leq i \leq n$, so $\lambda$ and $\mu$ are constant.

From (61), we have

$$
d(N-\lambda Y-\mu \xi)=0
$$

Therefore, we can find a constant vector $e \in R_{2}^{n+3}$ such that

$$
N-\lambda Y-\mu \xi=e .
$$

From (11) and (77), we get that

$$
\langle e, e\rangle=\mu^{2}-2 \lambda, \quad\langle Y, e\rangle=1 .
$$

To prove the theorem, we consider the following three cases.

Case 1. $e$ is light-like, that is, $\mu^{2}-2 \lambda=0$.

Case 2. $e$ is space-like, that is, $\mu^{2}-2 \lambda>0$.

Case 3. $e$ is time-like, that is, $\mu^{2}-2 \lambda<0$.

First, we consider Case 1 ; $e$ is light-like, that is, $\mu^{2}-2 \lambda=0$. Then, there exists a $T \in O(n+2,2)$ such that

$$
\bar{e}=(-1, \overrightarrow{0}, 1)=e T=(N-\lambda Y-\mu \xi) T .
$$

Let $\bar{x}: M^{n} \rightarrow R_{1}^{n+1}$ be a hypersurface that its conformal position vector is $\bar{Y}=Y T$, then $\bar{N}=N T, \bar{\xi}=\xi T$, and

$$
\bar{e}=\bar{N}-\lambda \bar{Y}-\mu \bar{\xi}
$$

Writing

$$
\begin{gathered}
\bar{Y}=e^{\bar{\tau}}\left(\frac{\langle\bar{x}, \bar{x}\rangle+1}{2}, \bar{x}, \frac{\langle\bar{x}, \bar{x}\rangle-1}{2}\right), \\
\bar{\xi}=-\bar{H}\left(\frac{\langle\bar{x}, \bar{x}\rangle+1}{2}, \bar{x}, \frac{\langle\bar{x}, \bar{x}\rangle-1}{2}\right)+\bar{y}_{n+1},
\end{gathered}
$$

then from

$$
\langle\bar{Y}, \bar{e}\rangle=1, \quad\langle\bar{\xi}, \bar{e}\rangle=-\mu,
$$

we obtain that

$$
e^{\bar{\tau}}=1, \quad \bar{H}=\mu .
$$

Since $\bar{Y}=((\langle\bar{x}, \bar{x}\rangle+1) / 2, \bar{x},(\langle\bar{x}, \bar{x}\rangle-1) / 2)$, then $g=$ $\langle d \bar{x}, d \bar{x}\rangle=\bar{I}$. From (24) we have

$$
\operatorname{tr}(A)=n \lambda=\frac{1}{2 n}\left(n^{2} \kappa-1\right)
$$

Since $\kappa_{M}=\kappa$, so the mean curvature and scalar curvature of hypersurface $\bar{x}$ are constant.

Next, we consider Case 2 ; $e$ is space-like, that is, $\mu^{2}-2 \lambda>$ 0 . Then, there exists a $T \in O(n+2,2)$ such that

$$
\bar{e}=\left(\overrightarrow{0}, \sqrt{\mu^{2}-2 \lambda}\right)=e T=(N-\lambda Y-\mu \xi) T
$$

Let $\bar{x}: M^{n} \rightarrow H_{1}^{n+1}(-1)$ be a hypersurface which its conformal position vector is $\bar{Y}=Y T$; then $\bar{N}=N T, \bar{\xi}=\xi T$, and

$$
\bar{e}=\bar{N}-\lambda \bar{Y}-\mu \bar{\xi}
$$

Writing $\bar{Y}=e^{\bar{\tau}}(\bar{x}, 1), \bar{\xi}=-\bar{H}(\bar{x}, 1)+\bar{y}_{n+1}$, then from

$$
\langle\bar{Y}, \bar{e}\rangle=1, \quad\langle\bar{\xi}, \bar{e}\rangle=-\mu,
$$

we obtain that

$$
e^{\bar{\tau}}=\frac{1}{\sqrt{\mu^{2}-2 \lambda}}, \quad \bar{H}=\mu .
$$

Since $\langle d \bar{x}, d \bar{x}\rangle=\left(\mu^{2}-2 \lambda\right) g$,

$$
\kappa_{M}=\frac{1}{\mu^{2}-2 \lambda} \kappa
$$

Therefore, the mean curvature and scalar curvature of hypersurface $\bar{x}$ are constant.

Finally, we consider Case 3 ; $e$ is time-like, that is, $\mu^{2}-2 \lambda<$ 0 . Then, there exists a $T \in O(n+2,2)$ such that

$$
\bar{e}=\left(-\sqrt{2 \lambda-\mu^{2}}, \overrightarrow{0}\right)=e T=(N-\lambda Y-\mu \xi) T .
$$

Let $\bar{x}: M^{n} \rightarrow S_{1}^{n+1}(1)$ be a hypersurface that its conformal position vector is $\bar{Y}=Y T$; then $\bar{N}=N T, \bar{\xi}=\xi T$, and

$$
\bar{e}=\bar{N}-\lambda \bar{Y}-\mu \bar{\xi} .
$$


Writing $\bar{Y}=e^{\bar{\tau}}(1, \bar{x}), \bar{\xi}=-\bar{H}(1, \bar{x})+\bar{y}_{n+1}$, then from

$$
\langle\bar{Y}, \bar{e}\rangle=1, \quad\langle\bar{\xi}, \bar{e}\rangle=-\mu,
$$

we obtain that

$$
e^{\bar{\tau}}=\frac{1}{\sqrt{2 \lambda-\mu^{2}}}, \quad \bar{H}=\mu .
$$

Since $\langle d \bar{x}, d \bar{x}\rangle=\left(2 \lambda-\mu^{2}\right) g$,

$$
\kappa_{M}=\frac{1}{2 \lambda-\mu^{2}} \kappa \text {. }
$$

Therefore, the mean curvature and scalar curvature of hypersurface $\bar{x}$ are constant. Thus, we complete the proof the Theorem 9 .

Corollary 10. Let $x: M^{n} \rightarrow M_{1}^{n+1}(c)$ be a space-like hypersurface without umbilical points. If conformal invariants of $x$ satisfy

$$
\text { (1) }, C=0, \quad \text { (2) }, A=\lambda g \text {, }
$$

then $x$ is conformal equivalent to a maximal space-like hypersurface with constant scalar curvature in $H_{1}^{n+1}(-1)$.

Proof. From the process of proof of Theorem 9, we have that $x$ is conformal equivalent to a maximal space-like hypersurface with constant scalar curvature. Next, we prove that $x$ is a maximal space-like hypersurface with constant scalar curvature in $H_{1}^{n+1}(-1)$. In fact, using covariant derivative of $\nabla B$ and $C=0$, we have

$$
\begin{aligned}
\frac{1}{2} \Delta|B|^{2}= & \sum_{i j k}\left|B_{i j, k}\right|^{2}+\sum_{i j} \Delta B_{i j} \\
= & \sum_{i j k}\left|B_{i j, k}\right|^{2}+\left(\frac{n-1}{n}\right)^{2}+\frac{(n-1)}{n} \operatorname{tr}(A) \\
& +n \sum_{i j k} B_{i k} B_{k j} A_{j i} .
\end{aligned}
$$

Since $|B|^{2}=(n-1) / n$ and $A=\lambda g$, we get that

$$
\sum_{i j k}\left|B_{i j, k}\right|^{2}+\left(\frac{n-1}{n}\right)^{2}+2(n-1) \lambda=0 .
$$

Thus, $\lambda \leq-(n-1) / 2 n^{2}<0$. From the process of proof of the Theorem 9, we have that $x$ is conformal equivalent to a maximal space-like hypersurface with constant scalar curvature in $H_{1}^{n+1}(-1)$.

\section{Some Special Willmore Space-Like Hypersurfaces}

In this section, we consider some special Willmore hypersurfaces. First, we give an example of the Willmore spacelike hypersurface with constant mean curvature and constant scalar curvature in $H_{1}^{n+1}(-1)$.
Example 11. Let

$$
\begin{aligned}
H^{k}\left(r_{1}\right) & =\left\{u=\left(u_{0}, \vec{u}_{1}\right) \in R_{1}^{k+1} \mid\langle u, u\rangle=-r_{1}^{2}\right\}, \\
H^{n-k}\left(r_{2}\right) & =\left\{v=\left(v_{0}, \vec{v}_{1}\right) \in R_{1}^{n-k+1} \mid\langle v, v\rangle=-r_{2}^{2}\right\},
\end{aligned}
$$

where $r_{1}^{2}+r_{2}^{2}=1$. Define space-like hypersurface

$$
\begin{gathered}
x: H^{k}\left(r_{1}\right) \times H^{n-k}\left(r_{2}\right) \longrightarrow H_{1}^{n+1}(-1), \\
x(u, v)=\left(u_{0}, v_{0}, \vec{u}_{1}, \vec{v}_{1}\right) \in H_{1}^{n+1}(-1) \subset R_{2}^{n+2} .
\end{gathered}
$$

Then, unit normal vector of $x$ is $e_{n+1}=\left(\left(r_{2} / r_{1}\right) u_{0},-\left(r_{1} / r_{2}\right) v_{0}\right.$, $\left.\left(r_{2} / r_{1}\right) \vec{u}_{1},-\left(r_{1} / r_{2}\right) \vec{v}_{1}\right)$; thus, the induced metric and the second fundamental form of $x$ are, respectively,

$$
\begin{gathered}
I=I_{u}+I_{v}, \quad I I=\frac{r_{2}}{r_{1}} I_{u}-\frac{r_{1}}{r_{2}} I_{v}, \\
n H=k \frac{r_{2}}{r_{1}}-(n-k) \frac{r_{1}}{r_{2}}, \\
|I I|^{2}=k\left(\frac{r_{2}}{r_{1}}\right)^{2}+(n-k)\left(\frac{r_{1}}{r_{2}}\right)^{2} .
\end{gathered}
$$

Now, we assume that $x$ is Willmore. Since $H$ and $|I I|^{2}$ are constant, from (33), we get that the Euler-Lagrange equation (51) is

$$
k\left(\frac{r_{2}}{r_{1}}\right)^{6}+(3 k-2 n)\left(\frac{r_{2}}{r_{1}}\right)^{4}+(3 k-n)\left(\frac{r_{2}}{r_{1}}\right)^{2}+k=0 .
$$

Since $r_{1}^{2}+r_{2}^{2}=1$, we get that

$$
r_{1}=-\sqrt{\frac{n-k}{n}}, \quad r_{2}=-\sqrt{\frac{k}{n}} .
$$

Corollary 12. Space-like hypersurface $x: H^{k}\left(r_{1}\right) \times$ $H^{n-k}\left(r_{2}\right) \rightarrow H_{1}^{n+1}(-1)$ is Willmore if and only if

$$
r_{1}=-\sqrt{\frac{n-k}{n}}, \quad r_{2}=-\sqrt{\frac{k}{n}} .
$$

Remark 13. Space-like hypersurface $x: H^{k}\left(r_{1}\right) \times H^{n-k}\left(r_{2}\right) \rightarrow$ $H_{1}^{n+1}(-1)$ is maximal if and only if

$$
r_{1}=-\sqrt{\frac{k}{n}}, \quad r_{2}=-\sqrt{\frac{n-k}{n}} .
$$

Thus, maximal hypersurfaces are not Willmore in general.

Theorem 14. Let $x: M^{n} \rightarrow M_{1}^{n+1}(c)$ be a Willmore space-like hypersurface without umbilical points. If conformal invariants of $x$ satisfy

$$
\text { (1), } C=0, \quad \text { (2), } A=\mu B+\lambda g \text {, }
$$


then $x$ is conformal equivalent to the space-like hypersurface in $H_{1}^{n+1}(-1)$ with constant mean curvature and constant scalar curvature, and

$$
2|h|^{2} H=\operatorname{tr}\left(h^{3}\right)+n H^{3}, \quad|h|^{2}-n H^{2} \leq n,
$$

where $h=\left(h_{i j}\right)$. If $|h|^{2}-n H^{2}=n$, then $x$ is

$$
H^{k}\left(-\sqrt{\frac{n-k}{n}}\right) \times H^{n-k}\left(-\sqrt{\frac{k}{n}}\right) .
$$

Proof. Let $x: M^{n} \rightarrow M_{1}^{n+1}(c)$ be a Willmore space-like hypersurface without umbilical points. Let $\left\{E_{1}, \ldots, E_{n}\right\}$ be the local orthonormal basis for $g$ such that

$$
\left(B_{i j}\right)=\operatorname{diag}\left(b_{1}, \ldots, b_{n}\right) \text {, }
$$

and corresponding principal curvatures are $\left(k_{1}, \ldots, k_{2}\right)$. Since

$$
\text { (1), } C=0, \quad(2), A=\mu B+\lambda g,
$$

and since $x$ is Willmore, from (51), we get that

$$
\frac{n-1}{n} \mu=\sum_{i} b_{i}^{3}
$$

From (33), we have

$$
2|h|^{2} H=\operatorname{tr}\left(h^{3}\right)+n H^{3} .
$$

By calculation of $(1 / 2) \Delta|h|^{2}$, we have

$$
\begin{aligned}
\frac{1}{2} \Delta|h|^{2}= & \sum_{i j k}\left|h_{i j, k}\right|^{2}-n^{2}|\nabla H|^{2}+\frac{1}{2} \sum_{i j} R_{i j i j}\left(k_{i}-k_{j}\right)^{2} \\
= & \sum_{i j k}\left|h_{i j, k}\right|^{2}-n^{2}|\nabla H|^{2}+n c|h|^{2}-n^{2} c H^{2}+|h|^{4} \\
& -n H \sum_{i} k_{i}^{3} .
\end{aligned}
$$

Since (1), $C=0,(2), A=\mu B+\lambda g$, so $x$ is conformal equivalent to a space-like hypersurface with constant mean curvature and constant scalar curvature. We can assume that mean curvature and scalar curvature of $x$ are constant. Thus, from the Gauss equation of $x$, we get that

$$
|h|^{2}=\text { constant }, \quad H=\text { constant. }
$$

From (112), we have

$$
0=\sum_{i j k}\left|h_{i j, k}\right|^{2}+n c|h|^{2}-n^{2} c H^{2}+|h|^{4}-n H \sum_{i} k_{i}^{3} .
$$

Combining (111) and (114), we get that

$$
\sum_{i j k}\left|h_{i j, k}\right|^{2}+n c\left(|h|^{2}-n H^{2}\right)+\left(|h|^{2}-n H^{2}\right)^{2}=0 .
$$

Since $|h|^{2}-n H^{2} \geq 0$ and from (115), we get that

$$
c=-1 \text {. }
$$

Thus, $x$ is a space-like hypersurface in $H_{1}^{n+1}(-1)$. From (115), we have

$$
\sum_{i j k}\left|h_{i j, k}\right|^{2}+\left(|h|^{2}-n H^{2}\right)\left(|h|^{2}-n H^{2}-n\right)=0 .
$$

Since $|h|^{2}-n H^{2} \geq 0$, from (117), we have

$$
|h|^{2}-n H^{2} \leq n
$$

If $|h|^{2}-n H^{2}=n$, then $h_{i j, k}=0$; thus, the principal curvatures of $x$ are constant. It is well known that space-like isoparametric hypersurfaces in $H_{1}^{n+1}(-1)$ are either totally umbilical hypersurfaces or $H^{k}\left(r_{1}\right) \times H^{n-k}\left(r_{2}\right)$ (see [11]).

Thus, we complete the proof of Theorem 14 .

\section{Acknowledgments}

Tongzhu Li is partially supported by Grant no. 10801006 of NSFC; Changxiong Nie is supported by Grant no. 1097055 of NSFC.

\section{References}

[1] C. Wang, "Moebius geometry of submanifolds in $\mathbb{S}^{n}$," Manuscripta Mathematica, vol. 96, no. 4, pp. 517-534, 1998.

[2] M. A. Akivis and V. V. Goldberg, "A conformal differential invariant and the conformal rigidity of hypersurfaces," Proceedings of the American Mathematical Society, vol. 125, no. 8, pp. 2415-2424, 1997.

[3] H. Li, "Willmore hypersurfaces in a sphere," Asian Journal of Mathematics, vol. 5, no. 2, pp. 365-377, 2001.

[4] H. Li, "Willmore surfaces in $S^{n}$," Annals of Global Analysis and Geometry, vol. 21, no. 2, pp. 203-213, 2002.

[5] H. Z. Li, H. L. Liu, C. P. Wang, and G. S. Zhao, "Möbius isoparametric hypersurfaces in $\mathbb{S}^{n+1}$ with two distinct principal curvatures," Acta Mathematica Sinica, vol. 18, no. 3, pp. 437-446, 2002.

[6] H. Li and C. Wang, "Möbius geometry of hypersurfaces with constant mean curvature and scalar curvature," Manuscripta Mathematica, vol. 112, no. 1, pp. 1-13, 2003.

[7] C. X. Nie, Conformal geometry of hypersurfaces and surfaces in Lorentz space forms [Ph.D. thesis], Peking University, 2006.

[8] M. P. Dussan and M. Magid, "Conformally flat Lorentzian hypersurfaces in the conformal compactification of Lorentz space," Journal of Geometry and Physics, vol. 57, no. 12, pp. 24662482, 2007.

[9] L. J. Alías and B. Palmer, "Zero mean curvature surfaces with non-negative curvature in flat Lorentzian 4-spaces," The Royal Society of London. Series A, vol. 455, no. 1982, pp. 631-636, 1999.

[10] M. Barros, A. Ferrández, P. Lucas, and M. A. Meroño, "Willmore tori and Willmore-Chen submanifolds in pseudoRiemannian spaces," Journal of Geometry and Physics, vol. 28, no. 1-2, pp. 45-66, 1998.

[11] M. A. Magid, "Lorentzian isoparametric hypersurfaces," Pacific Journal of Mathematics, vol. 118, no. 1, pp. 165-197, 1985. 


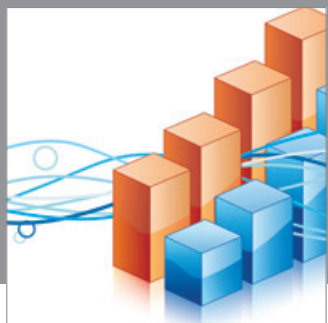

Advances in

Operations Research

mansans

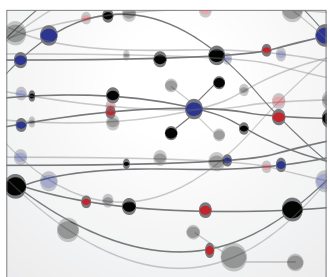

The Scientific World Journal
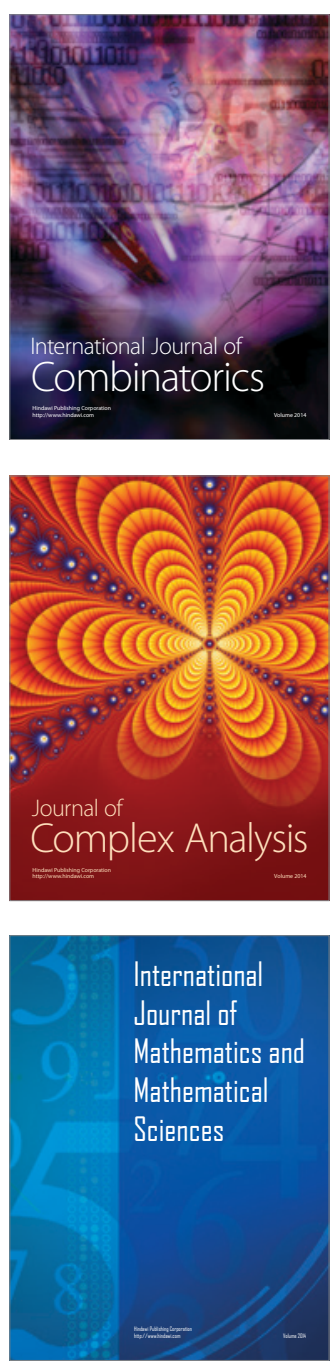
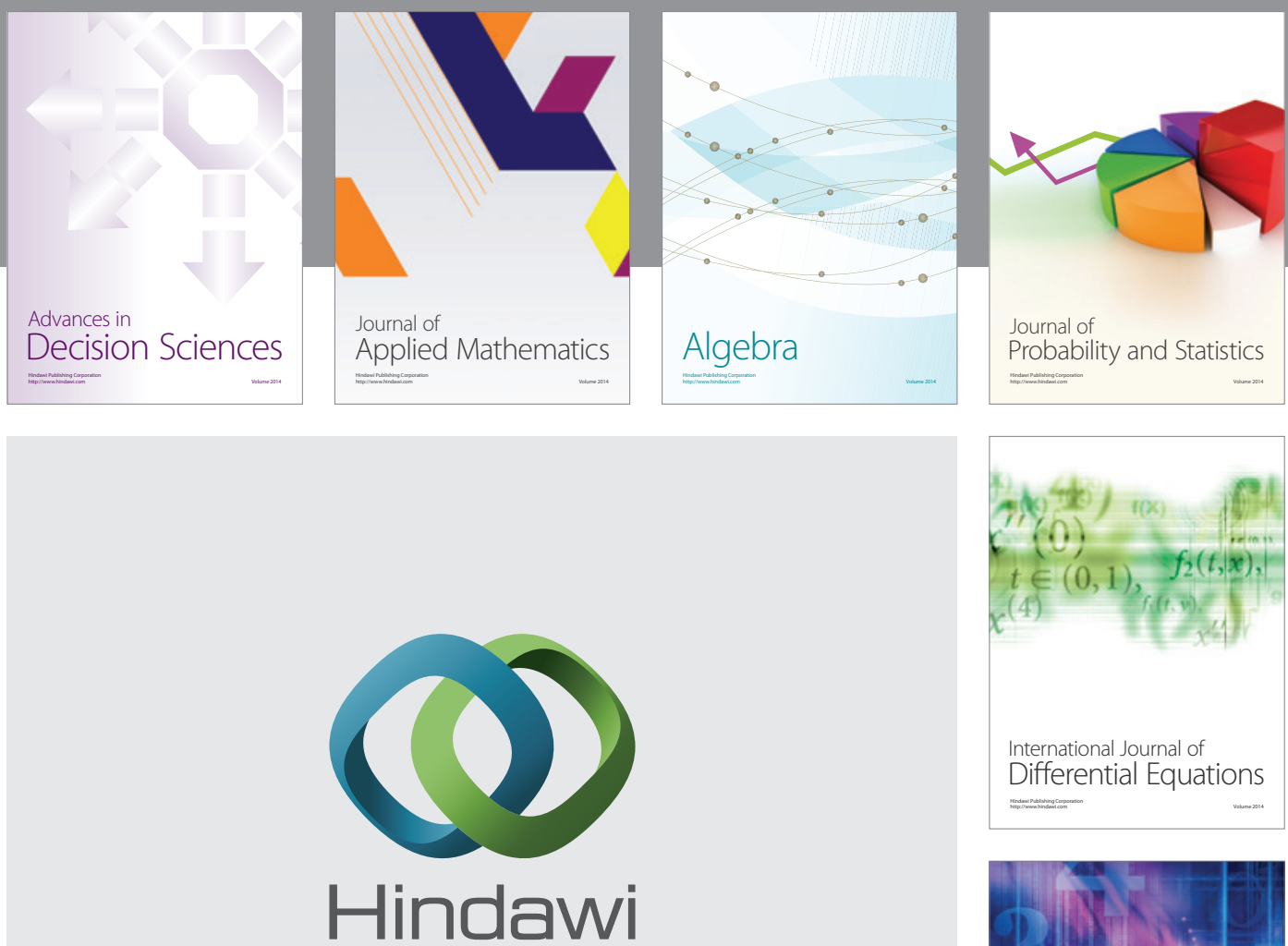

Submit your manuscripts at http://www.hindawi.com
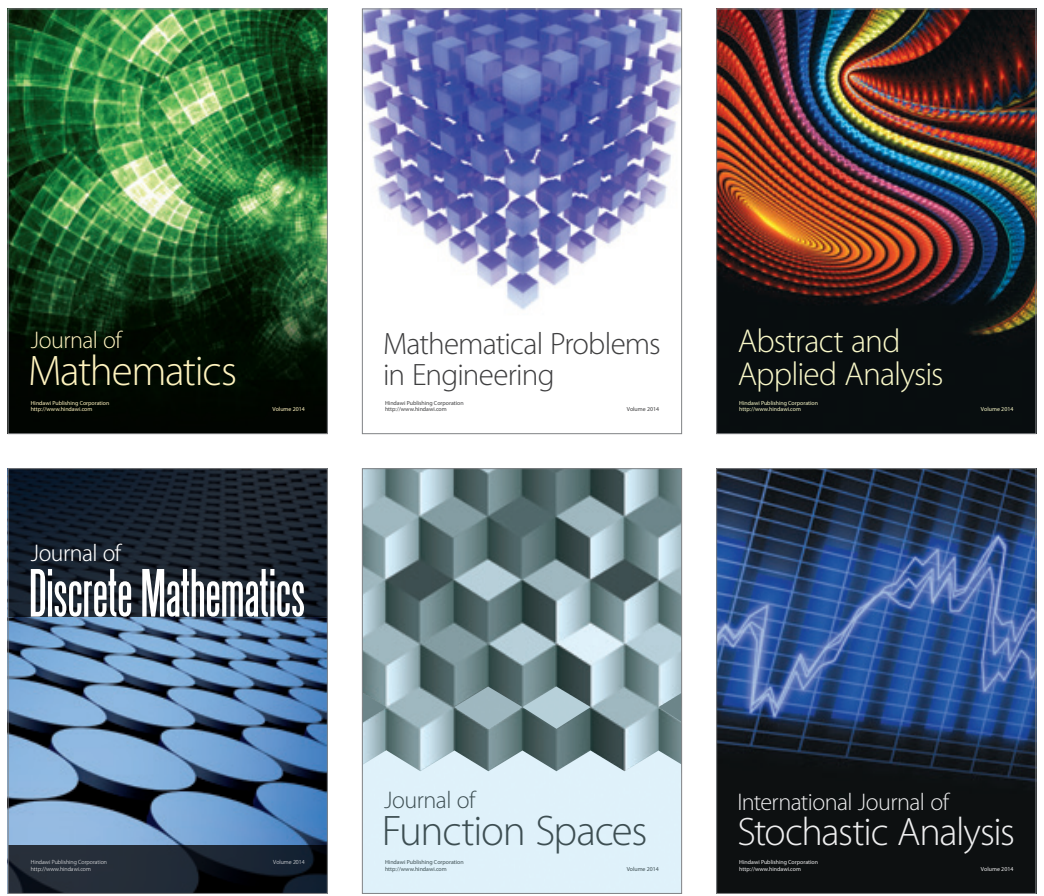

Journal of

Function Spaces

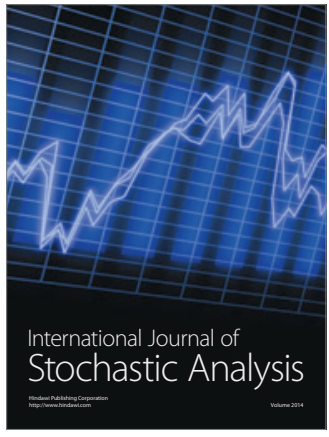

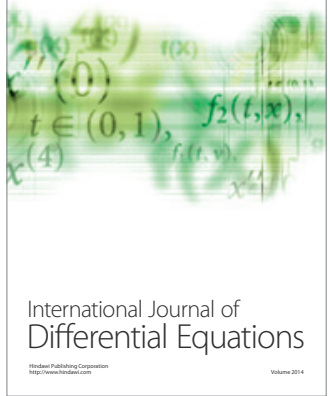
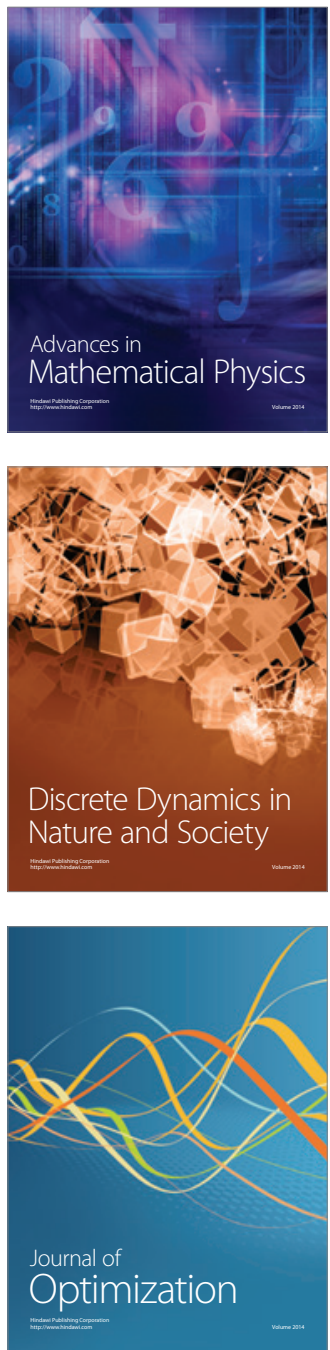